1 Instituto de Medicina Integral Professor Fernando

Figueira (Imip), Grupo de Estudos de Gestão e Avaliação em Saúde (Geas) - Recife (PE), Brasil. natchallya@hotmail.com

\section{Vigilância em Saúde regionalizada em Pernambuco: um estudo de caso}

\author{
Regionalized Health Surveillance in the State of Pernambuco: a case \\ study
}

Natália Barros Lopes', Ana Coelho de Albuquerque', Eronildo Felisberto'

DOI: 10.1590/0103-1104201912205

RESUMO O objetivo deste estudo foi o de avaliar a implantação da Vigilância em Saúde (VS) regionalizada em Pernambuco. Trata-se de um estudo avaliativo realizado nas etapas normativa e exploratória. A avaliação normativa utilizou abordagem quantitativa para as dimensões estrutura e processo, medindo o grau de implantação da VS em quatro regiões de saúde de Pernambuco. A etapa exploratória consistiu na realização de entrevistas semiestruturadas com informantes-chave para apreender a percepção desses indivíduos acerca do processo de regionalização da VS em Pernambuco e dos fatores facilitadores e dificultadores desse processo. Nas regiões I, II e III, a VS foi classificada como 'parcialmente implantada' e na região IV, como 'implantada'. A 'estrutura' foi classificada como 'parcialmente implantada' em todas as regiões, e o 'processo' foi classificado como 'parcialmente implantado' nas regiões I e III, e como 'implantado' nas regiões II e IV. Conclui-se que a regionalização da VS em Pernambuco ainda está incompleta apesar de ser um processo de grande relevância, uma vez que o nível regional pode planejar e promover estratégias mais adequadas para cada contexto, privilegiando as diferentes realidades dos municípios.

PALAVRAS-CHAVE Regionalização. Vigilância em saúde pública. Avaliação em saúde.

ABSTRACT This study aimed to evaluate the implementation of Regionalized Health Surveillance (HS) in the State of Pernambuco. It is an evaluative study carried out by means of the normative and the exploratory steps. The normative evaluation applied a quantitative approach for the structure and process dimensions, measuring the degree of HS implementation in four health regions of Pernambuco. The exploratory step consisted of semi-structured interviews with key informants so to apprehend also their perception about the HS regionalization process in Pernambuco as the factors that ease and hinder the process. HS was classified as 'partially implemented' in regions I, II and III, and 'implemented' in region IV. 'Structure' was classified as 'partially implemented' in all regions. 'Process' was classified as 'partially implemented' in regions I and III, and 'implemented' in regions II and IV. We concluded that HS regionalization in Pernambuco is still incomplete, despite being a process of great relevance, since the regional level is able to plan and promote strategies more appropriate for each context, given the specific characteristics of each municipality.

KEYWORDS Regional health planning. Public health surveillance. Health evaluation. 


\section{Introdução}

A descentralização das ações e serviços de saúde foi definida como um dos princípios organizativos do Sistema Único de Saúde (SUS), tendo como base, inicialmente, a municipalização e, posteriormente, a ênfase na regionalização' ${ }^{\mathbf{1}}$ Essa organização surgiu como resposta à estrutura anterior ${ }^{2}$, de caráter centralizador e inadequado para um país do tamanho e da complexidade do Brasil ${ }^{3}$. No entanto, destaca-se que a fragilidade no planejamento das estratégias de descentralização do SUS comprometeu sua adequação às múltiplas realidades brasileiras. Com isso, não houve diversificação de políticas e investimentos que se adequassem às diversas condições políticas, administrativas, técnicas, financeiras e de necessidades de saúde identificadas nos municípios, visando à redução das desigualdades ${ }^{\mathbf{4}}$.

Esses fatores foram determinantes para a inflexão da condução política do SUS no sentido da regionalização. Porém, segundo o estudo realizado por Vargas et al. ${ }^{5}$, a regionalização apresenta baixa implantação e desenvolvimento no Brasil, pois depende de responsabilidades complexas da entidade regional e da fragilizada corresponsabilização dos níveis estadual e federal. Assim, com o objetivo de reestruturar e fortalecer o SUS de forma a promover com maior eficiência as ações de saúde de maneira regionalizada e para que o cidadão possa conhecer os serviços ofertados pelas regiões de saúde, foi publicado o Decreto $\mathrm{n}^{0} 7.508$ de 28 de junho de 2011, que regulamentou a Lei Orgânica da Saúde $n^{\circ} 8.080 / 90^{6}$.

Vale ressaltar que características socioeconômicas, culturais e ambientais, entre outras, interferem no risco de ocorrência de determinados agravos à saúde, muitas vezes, ultrapassando os limites dos municípios e, dessa maneira, as ações de Vigilância em Saúde (VS) devem ser desenvolvidas de forma a que sejam planejadas regionalmente e executadas de maneira articulada ${ }^{7}$. Assim, a operacionalização da VS requer a valorização dos princípios de regionalização e hierarquização dos serviços a partir da delimitação das áreas de abrangência e de influência dos serviços conforme sua capacidade operacional, além do mapeamento da região em microáreas de risco, definidas com base no perfil epidemiológico da população ${ }^{8}$.

Portanto, para a execução das ações de VS de maneira regionalizada são necessários estrutura adequada e processos de trabalho bem definidos, condições primordiais para o alcance dos indicadores pactuados, o que viabiliza a qualidade da gestão no SUS. A importância da VS como política de saúde capaz de intervir nos problemas sanitários da população e o seu papel na conformação da regionalização no Brasil8 justificam a realização desta pesquisa. A implementação do SUS exige cada vez mais o uso de ferramentas que possibilitem a identificação dos principais problemas de saúde 9 , não sendo diferente na área da VS.

Dessa forma, este estudo tem como objetivo avaliar a implantação da VS regionalizada no estado de Pernambuco e identificar os fatores favoráveis e desfavoráveis ao processo de regionalização.

\section{Material e métodos}

Trata-se de estudo avaliativo realizado nas etapas normativa e uma exploratória. A avaliação normativa utilizou abordagem quantitativa para as dimensões estrutura e processo, com a medição do grau de implantação da VS em quatro regiões de saúde de Pernambuco, as quais figuram como sedes das quatro macrorregionais de saúde do estado. Para tanto, foi utilizada a abordagem de estrutura e processo proposta por Donabedian para se analisar em que medida a intervenção está implantada de modo a alcançar efetivamente os objetivos propostos ${ }^{10}$.

A etapa exploratória consistiu na realização de entrevistas semiestruturadas com quatro informantes-chave no nível regional (GR) e com três gestores do nível central (GC) da Secretaria Executiva de Vigilância em Saúde 
de Pernambuco (Sevs-PE). O objetivo foi apreender a percepção desses indivíduos acerca do processo de regionalização da VS em Pernambuco e dos fatores facilitadores e dificultadores desse processo.

Este estudo foi realizado nas quatro macrorregionais de saúde de Pernambuco - Recife, Caruaru, Serra Talhada e Petrolina -, arranjos territoriais que agregam mais de uma região de saúde, com o objetivo de organizar, entre si, ações e serviços de média complexidade, i.e., procedimentos e ações que requerem maior tecnologia, que apresentam oferta escassa no estado e cuja demanda requer agregação, ou formação de escala, e de alta complexidade, complementando, desse modo, a atenção à saúde das populações desses territórios ${ }^{11}$.

A avaliação normativa foi desenvolvida em quatro etapas.

\section{Etapa 1- Elaboração do modelo lógico da VS regionalizada}

Para descrever a intervenção avaliada, construiu-se o Modelo Lógico (ML) da VS regionalizada, inicialmente desenvolvido com base em análise documental que utilizou informações contidas em documentos oficiais e portarias que regulamentam a VS (quadro 1).

Quadro 1. Descrição das Portarias/Instruções Normativas utilizadas para construção do Modelo Lógico da Vigilância em Saúde regionalizada de Pernambuco

\begin{tabular}{|c|c|}
\hline Portaria/Instrução Normativa & Descrição \\
\hline $\begin{array}{l}\text { Portaria no } 1.399 \text { de } 15 \text { de dezembro } \\
\text { de } 1999 \mathbf{1 2}\end{array}$ & $\begin{array}{l}\text { Regulamenta a NOB SUS 01/96 - Competências da União, estados, municííos e } \\
\text { Distrito Federal, na área de epidemiologia e controle de doenças, define a sistemática } \\
\text { de financiamento e dá outras providências. }\end{array}$ \\
\hline $\begin{array}{l}\text { Portaria no } 1.378 \text {, de } 9 \text { de julho de } \\
2013^{13}\end{array}$ & $\begin{array}{l}\text { Regulamenta as responsabilidades e define diretrizes para execução e financiamento } \\
\text { das ações de Vigilância em Saúde pela União, estados, Distrito Federal e municípios, } \\
\text { relativos ao Sistema Nacional de Vigilância em Saúde e Sistema Nacional de Vigilân- } \\
\text { cia Sanitária. }\end{array}$ \\
\hline $\begin{array}{l}\text { Decreto no } 7.508 \text {, de } 28 \text { de junho de } \\
20116\end{array}$ & $\begin{array}{l}\text { Regulamenta a Lei no 8.080, de } 19 \text { de setembro de 1990, para dispor sobre a orga- } \\
\text { nização do Sistema Único de Saúde - SUS, o planejamento da saúde, a assistência à } \\
\text { saúde e a articulação interfederativa, e dá outras providências. }\end{array}$ \\
\hline $\begin{array}{l}\text { Diretrizes Nacionais da Vigilância em } \\
\text { Saúde (2010)(14 }\end{array}$ & $\begin{array}{l}\text { Trata das diretrizes gerais e estratégicas da Vigilância em Saúde e aborda questões } \\
\text { relativas ao enfrentamento das Emergências em Saúde Pública. }\end{array}$ \\
\hline $\begin{array}{l}\text { Portaria no 20, de } 03 \text { de outubro de } \\
2003^{15}\end{array}$ & $\begin{array}{l}\text { Regulamenta a coleta de dados, fluxo e periodicidade de envio das informações sobre } \\
\text { óbitos e nascidos vivos para os Sistemas de Informações em Saúde - SIM e Sistema } \\
\text { de Informação sobre Nascidos Vivos - Sinasc. }\end{array}$ \\
\hline $\begin{array}{l}\text { Instrução Normativa no } 02 \text { de } 22 \text { de } \\
\text { novembro de } 2005^{16}\end{array}$ & $\begin{array}{l}\text { Regulamenta as atividades da vigilância epidemiológica com relação à coleta, fluxo e a } \\
\text { periodicidade de envio de dados da notificação compulsória de doenças por meio do } \\
\text { Sistema de Informação de Agravos de Notificação - Sinan. }\end{array}$ \\
\hline
\end{tabular}

Fonte: Elaboração própria.

Posteriormente, o ML foi submetido por meio eletrônico a sete especialistas da área da VS e da avaliação em saúde, os quais contribuíram com a supressão ou inclusão dos itens do modelo, finalizado após duas rodadas. O delineamento do ML final explicitou os componentes (i) Gestão, que composto de seis subcomponentes e (ii) Processo de Trabalho, que contou com cinco subcomponentes (quadro 2). 
Quadro 2. Modelo Lógico da Vigilância em Saúde regionalizada. Pernambuco, 2017

\begin{tabular}{|c|c|c|c|}
\hline Componentes & Subcomponentes & Processo & Impacto \\
\hline \multirow[t]{8}{*}{ Gestão } & Normas/Legislação & Existência de legislação atualizada & \multirow{18}{*}{ 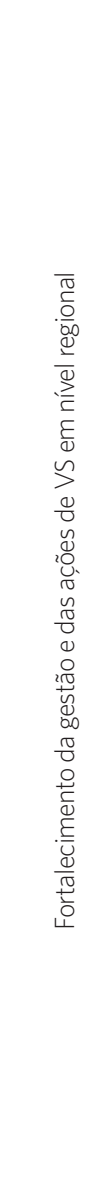 } \\
\hline & Capacidade Instalada & $\begin{array}{l}\text { Estrutura física, e recursos materiais adequados ao desenvolvimento } \\
\text { das ações de VS }\end{array}$ & \\
\hline & Recursos Financeiros & Utilização dos recursos financeiros para as ações de VS & \\
\hline & Recursos Humanos & Qualificação profissional e existência de organograma da VS & \\
\hline & Informação e comu- & Apoio técnico aos municípios na elaboração de informes e boletins & \\
\hline & & $\begin{array}{l}\text { Discussão sobre tema de VS nas reuniões da Comissão Intergestores } \\
\text { Regional (CIR) }\end{array}$ & \\
\hline & Vigilância Labora- & Processamento de amostras em nível regional & \\
\hline & & Apoio técnico aos municípios quanto às amostras laboratoriais & \\
\hline \multirow{10}{*}{$\begin{array}{l}\text { Processo de } \\
\text { trabalho }\end{array}$} & \multirow{2}{*}{$\begin{array}{l}\text { Sistemas de Infor- } \\
\text { mação }\end{array}$} & Alimentação do banco de dados dos Sistemas de Informação & \\
\hline & & Apoio operacional aos municípios quanto aos Sistemas de Informação & \\
\hline & \multirow[t]{2}{*}{ Planejamento } & Planejamento estratégico de ações & \\
\hline & & $\begin{array}{l}\text { Ações intersetoriais e ações conjuntas entre a Secretaria Executiva de } \\
\text { VS (nível central) e a VS regional }\end{array}$ & \\
\hline & \multirow[t]{2}{*}{ Integração das aç̃̃es } & Assessoramento permanente aos municípios & \\
\hline & & $\begin{array}{l}\text { Cooperação técnica entre a Secretaria Executiva de VS (nível central) e } \\
\text { a VS regional }\end{array}$ & \\
\hline & $\begin{array}{l}\text { Monitoramento e } \\
\text { avaliação }\end{array}$ & Monitoramento e discussão dos indicadores de VS & \\
\hline & \multirow[t]{3}{*}{$\begin{array}{l}\text { Desenvolvimento } \\
\text { das ações de VS }\end{array}$} & $\begin{array}{l}\text { Municípios estruturados para resposta aos eventos de importância em } \\
\text { saúde pública }\end{array}$ & \\
\hline & & Realização e divulgação de análises epidemiológicas & \\
\hline & & $\begin{array}{l}\text { Apoio aos municípios na investigação epidemiológica e nas ações de } \\
\text { promoção, prevenção e controle }\end{array}$ & \\
\hline
\end{tabular}

Fonte: Elaboração própria.

\section{Etapa 2 - Construção da matriz de indicadores e julgamento}

A partir do ML, elaborou-se a matriz de análise e julgamento, que também foi posteriormente submetida aos mesmos especialistas, em duas rodadas. Para a avaliação do grau de implantação, aplicou-se um questionário semiestruturado aos coordenadores de VS das quatro regiões selecionadas, elaborado com base no ML e da matriz.

O quadro 3 explicita a matriz construída com o emprego de seis critérios de estrutura, i.e., Normas e Legislação, Capacidade Instalada, Recursos Financeiros, Recursos Humanos, Informação e Comunicação, e Vigilância Laboratorial, num total de 25 indicadores. Empregou, também, cinco critérios de processo, i.e., Sistemas de Informação, Planejamento, Integração das ações, Monitoramento e avaliação, Desenvolvimento das ações em VS, num total de 18 indicadores. Os indicadores selecionados geraram o instrumento para coleta de dados. 
Quadro 3. Matriz de análise e julgamento da Vigilância em Saúde regionalizada. Pernambuco, 2017

\begin{tabular}{|c|c|c|c|c|c|}
\hline Componente & Critério & Indicadores & Padrão & $\begin{array}{l}\text { Fonte de } \\
\text { verificação }\end{array}$ & $\begin{array}{l}\text { Pontuação } \\
\text { máxima }\end{array}$ \\
\hline \multirow{25}{*}{ 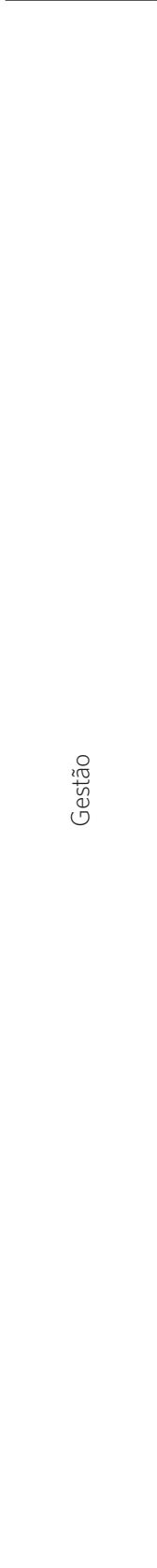 } & \multirow[t]{2}{*}{$\begin{array}{l}\text { Normas e } \\
\text { Legislação }\end{array}$} & $\begin{array}{l}\text { Existência de legislação atualizada às necessidades do contexto epidemio- } \\
\text { lógico. }\end{array}$ & $\operatorname{Sim}$ & $\begin{array}{l}\text { EIC/documen- } \\
\text { tos }\end{array}$ & 2 \\
\hline & & Divulgação de normas e portarias para os municípios da regional. & $\operatorname{Sim}$ & EIC & 1 \\
\hline & \multirow{10}{*}{$\begin{array}{l}\text { Capacidade } \\
\text { Instalada }\end{array}$} & Considera a quantidade de profissionais de nível superior suficiente. & $\operatorname{Sim}$ & $\mathrm{EIC}$ & 3 \\
\hline & & $\begin{array}{l}\text { Considera a qualidade técnica dos Recursos Humanos }(\mathrm{RH}) \text { suficiente para } \\
\text { cumprimento das atividades essenciais. }\end{array}$ & $\operatorname{Sim}$ & $\mathrm{EIC}$ & 3 \\
\hline & & $\begin{array}{l}\text { Considera o número de salas disponíveis para VS suficiente para alojar adequa- } \\
\text { damente as equipes. }\end{array}$ & $\operatorname{Sim}$ & EIC & 2 \\
\hline & & № de computadores disponíveis e em funcionamento para VS. & 1 por técnico & $\mathrm{EIC}$ & 2 \\
\hline & & № de impressoras disponíveis para VS. & $\begin{array}{l}2 \text { impres- } \\
\text { soras }\end{array}$ & $\mathrm{EIC}$ & 2 \\
\hline & & № de ramais disponíveis para VS. & 2 ramais & $\mathrm{EIC}$ & 2 \\
\hline & & Existência de internet disponível para VS. & $\operatorname{Sim}$ & EIC & 2 \\
\hline & & № veículos disponíveis para VS. & 4 veículos & EIC & 2 \\
\hline & & Considera o quantitativo de veículos suficiente em quantidade e qualidade. & $\mathrm{Sim}$ & $\mathrm{EIC}$ & 2 \\
\hline & & $\begin{array}{l}\text { Existência de recebimento e distribuição de materiais e insumos em quantitativo } \\
\text { suficiente para os municípios. }\end{array}$ & $\operatorname{Sim}$ & $\mathrm{EIC}$ & 1 \\
\hline & \multirow[t]{2}{*}{$\begin{array}{l}\text { Recursos } \\
\text { Financeiros }\end{array}$} & $\begin{array}{l}\text { Utilização do repasse da Sevs para a regional exclusivamente para as ações } \\
\text { de VS. }\end{array}$ & $\operatorname{Sim}$ & $\mathrm{EIC}$ & 2 \\
\hline & & Monitoramento bimestral da execução do repasse da Sevs pela regional. & $\operatorname{Sim}$ & $\mathrm{EIC}$ & 1 \\
\hline & \multirow{3}{*}{$\begin{array}{l}\text { Recursos } \\
\text { Humanos }\end{array}$} & \% adequado de profissionais qualificados na equipe de VS. & $100 \%$ & $\mathrm{EIC}$ & 3 \\
\hline & & Existência de organograma funcional na região de saúde, incorporando a VS. & $\operatorname{Sim}$ & $\mathrm{EIC}$ & 1 \\
\hline & & Oferece capacitações/treinamentos de VS para os municípios. & $\operatorname{Sim}$ & $\mathrm{EIC}$ & 1 \\
\hline & \multirow[t]{3}{*}{$\begin{array}{l}\text { Informação e } \\
\text { Comunicação }\end{array}$} & $\begin{array}{l}\text { \% de municípios apoiados tecnicamente na elaboração de seus informes/bole- } \\
\text { tins. }\end{array}$ & $100 \%$ & $\mathrm{EIC}$ & 2 \\
\hline & & Informes e boletins epidemiológicos elaborados e divulgados mensalmente. & $\operatorname{Sim}$ & EIC/in loco & 2 \\
\hline & & № de pautas da CIR com discussão de temas de VS. & $\begin{array}{l}1 \text { por bimes- } \\
\text { tre }\end{array}$ & $\begin{array}{l}\text { EIC/Pautas da } \\
\text { CIR }\end{array}$ & 1 \\
\hline & \multirow[t]{5}{*}{$\begin{array}{l}\text { Vigilância } \\
\text { Laboratorial }\end{array}$} & $\begin{array}{l}\text { Existência de processamento de amostras para diagnóstico clínico, ambiental e } \\
\text { bromatológico regionalmente. }\end{array}$ & $\begin{array}{l}\text { Sim, para } \\
\text { todos }\end{array}$ & $\mathrm{EIC}$ & 2 \\
\hline & & $\begin{array}{l}\text { Amostras provenientes dos municípios recebidas, armazenadas e transportadas } \\
\text { conforme demanda. }\end{array}$ & $\operatorname{Sim}$ & $\mathrm{EIC}$ & 2 \\
\hline & & $\begin{array}{l}\text { Laboratório regional com capacidade operacional adequada para análise da } \\
\text { qualidade da água. }\end{array}$ & $\operatorname{Sim}$ & EIC & 1 \\
\hline & & $\begin{array}{l}\text { Laboratório regional com capacidade operacional adequada para sorologias } \\
\text { (dengue, hepatites, chikungunya, doença de Chagas e Leishmaniose). }\end{array}$ & $\begin{array}{l}\text { Sim, para } \\
\text { todas }\end{array}$ & EIC & 2 \\
\hline & & Resultados laboratoriais acompanhados e divulgados junto aos municípios. & $\operatorname{Sim}$ & $\mathrm{EIC}$ & 1 \\
\hline \multirow{4}{*}{ 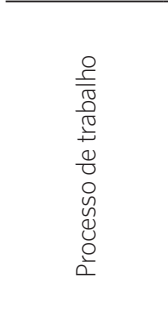 } & \multirow[t]{4}{*}{$\begin{array}{l}\text { Sistemas de } \\
\text { Informação }\end{array}$} & $\begin{array}{l}\text { Envio/transferência do banco de dados dos SIS para o nível central na periodici- } \\
\text { dade estabelecida. }\end{array}$ & $\operatorname{Sim}$ & $\begin{array}{l}\text { EIC/SIS (Nível } \\
\text { Central) }\end{array}$ & 2 \\
\hline & & \% de municípios com retroalimentação mensal dos dados. & $100 \%$ & $\begin{array}{l}\text { EIC/SIS (Nível } \\
\text { Central) }\end{array}$ & 2 \\
\hline & & $\begin{array}{l}\text { Análise de dados/indicadores operacionais dos municípios realizadas mensal- } \\
\text { mente. }\end{array}$ & $\operatorname{Sim}$ & $\mathrm{EIC}$ & 2 \\
\hline & & $\begin{array}{l}\text { Existência de assessoramento aos municípios na operacionalização dos siste- } \\
\text { mas conforme demanda. }\end{array}$ & $\operatorname{Sim}$ & $\mathrm{EIC}$ & 2 \\
\hline
\end{tabular}


Quadro 3. (cont.)

\begin{tabular}{|c|c|c|c|c|c|}
\hline \multirow{14}{*}{ 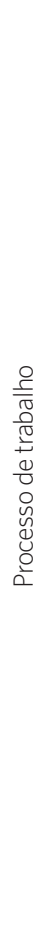 } & \multirow[t]{2}{*}{ Planejamento } & Realização de planejamento intersetorial. & $\geq 1$ plano & EIC & 1 \\
\hline & & Realização de planejamento baseados em análises de situação de saúde. & $\operatorname{sim}$ & $\mathrm{EIC}$ & 1 \\
\hline & \multirow{6}{*}{$\begin{array}{l}\text { Integração } \\
\text { (articulação) } \\
\text { das ações }\end{array}$} & № de reuniões entre as áreas técnicas da VS regional. & $\begin{array}{l}1 \text { reunião/ } \\
\text { mês }\end{array}$ & $\mathrm{EIC}$ & 1 \\
\hline & & № de reuniões entre a VS e a atenção à saúde regional. & $\begin{array}{l}1 \text { reunião/ } \\
\text { mês }\end{array}$ & $\mathrm{EIC}$ & 1 \\
\hline & & Ações conjuntas entre a VS regional e a Sevs, conforme demanda. & $\operatorname{Sim}$ & $\mathrm{EIC}$ & 2 \\
\hline & & \% de municípios assessorados quando as ações de VS. & $100 \%$ & $\mathrm{EIC}$ & 2 \\
\hline & & $\begin{array}{l}\text { \% de comitês e/ou grupos técnicos com participação conjunta (nível central e } \\
\text { nível regional). }\end{array}$ & $100 \%$ & $\mathrm{EIC}$ & 2 \\
\hline & & Realização de ações intersetoriais entre a VS e outros setores fora da saúde. & Sim & $\mathrm{EIC}$ & 1 \\
\hline & \multirow{2}{*}{$\begin{array}{l}\text { Monito- } \\
\text { ramento e } \\
\text { avaliação }\end{array}$} & Monitoramento trimestral dos indicadores de VS pela equipe regional. & $\operatorname{Sim}$ & $\mathrm{EIC}$ & 2 \\
\hline & & $\begin{array}{l}\text { Existência de reuniões para a discussão dos resultados do monitoramento das } \\
\text { ações de VS com os municípios. }\end{array}$ & $\operatorname{Sim}$ & $\mathrm{EIC}$ & 1 \\
\hline & \multirow{4}{*}{$\begin{array}{l}\text { Desenvolvi- } \\
\text { mento das } \\
\text { ações em VS }\end{array}$} & $\begin{array}{l}\text { Considera a VS da regional estruturada para detecção e resposta aos eventos de } \\
\text { importância para saúde pública. }\end{array}$ & $\operatorname{Sim}$ & $\mathrm{EIC}$ & 2 \\
\hline & & Análises epidemiológicas realizadas e divulgadas aos municípios mensalmente. & $\operatorname{Sim}$ & $\mathrm{EIC} /$ in loco & 2 \\
\hline & & $\begin{array}{l}\text { \% de municípios com apoio técnico da VS regional na investigação de casos } \\
\text { notificados, surtos e óbitos. }\end{array}$ & $100 \%$ & $\mathrm{EIC}$ & 2 \\
\hline & & $\begin{array}{l}\text { \% de municípios com apoio técnico da VS regional para ações de Promoção, } \\
\text { Prevenção e Controle de doenças e agravos. }\end{array}$ & $100 \%$ & $\mathrm{EIC}$ & 2 \\
\hline
\end{tabular}

Fonte: Elaboração própria.

EIC - Entrevista com Informante Chave.

SIS - Sistemas de Informação em Saúde.

Os dados primários foram coletados no período de agosto a setembro de 2017 por meio de entrevistas semiestruturadas aplicadas aos coordenadores de VS das quatro regiões selecionadas.

\section{Etapa 3 - Classificação do grau de implantação}

Para definição do grau de implantação, utilizaram-se indicadores de estrutura e processo de cada componente. Para a componente 'Gestão', foram atribuídos 45 pontos, e para 'Processo de Trabalho', 30 pontos. O grau de implantação foi estimado com base nos critérios e indicadores que compõem a matriz de julgamento. Os critérios utilizados para julgamento do grau de implantação da intervenção consideraram a proporção de adesão aos indicadores propostos. Consideraramse os seguintes critérios para julgamento do grau de implantação: implantado ( $\geq 80 \%)$; parcialmente implantado (51\% a $79 \%)$; e não implantado ( $\geq 50 \%)$. A determinação do grau de implantação permitiu classificar as regiões de saúde de Pernambuco em distintos níveis de implantação da VS.

\section{Percepção dos gestores}

Elaborou-se um roteiro de entrevista, aplicado a três gestores do nível central (GC1, GC2 e GC3) e aos coordenadores do nível regional (GR1, GR2, GR3 e GR4) com a finalidade de descrever como se deu o processo de regionalização da VS em Pernambuco, destacando-se as fragilidades e potencialidades desse processo.

A análise dos dados oriundos das entrevistas 
foi baseada na técnica de análise de conteúdo que se apoia na codificação da informação em categorias, de forma a dar sentido ao material estudado. Essa análise de conteúdo foi composta pelas fases de (i) pré-análise, que abrangeu a escolha do material, a formulação de hipóteses e objetivos e a elaboração de indicadores para a interpretação dos resultados; (ii) análise do material, que consistiu na codificação, categorização e quantificação da informação; e (iii) tratamento dos resultados, que envolveu procedimentos diversos ${ }^{17}$.

Após a leitura exaustiva do material utilizado como fonte documental, identificaram-se fatos ou informações que se relacionaram com fragilidades ou potencialidades do processo de regionalização da VS em Pernambuco. Realizou-se análise do tipo temática que, segundo Minayo ${ }^{\mathbf{1 8}}$, consiste em destacar os núcleos de sentido de uma comunicação, cuja presença ou repetição signifiquem algo para o objetivo analítico estudado. Os dados considerados relevantes foram inseridos em uma matriz de análise de forma ordenada e cronológica.

O estudo foi aprovado pelo Comitê de Ética em Pesquisa em Seres Humanos do Instituto de Medicina Integral Professor Fernando Figueira (Imip) sob o parecer $\mathrm{n}^{\circ} 2.134 .516 \mathrm{de}$ 23 de junho de 2017.

\section{Resultados}

\section{Grau de implantação da VS}

A VS nas regiões I, II e III foi classificada 'parcialmente implantada' (64,0\%, 76,0\% e $61,3 \%$ respectivamente) e, na região IV, 'implantada' (82,6\%). A 'estrutura' foi classificada 'parcialmente implantada' em todas as regiões (66,6\%, 71,1\%, 57,7\% e 77,7\%, respectivamente) (tabela 1). Já o 'processo', foi classificado 'parcialmente implantado' nas regiões I e III (60,0\% e 66,6\%) e 'implantado' nas regiões II e IV (83,3\% e 90,0\%) (tabela 2$)$.

\begin{tabular}{|c|c|c|c|c|c|c|}
\hline $\begin{array}{l}\text { Subcompo- } \\
\text { nente }\end{array}$ & Indicador & $\begin{array}{r}\text { Pontuação } \\
\text { máxima }\end{array}$ & RI & RII & RIII & RIV \\
\hline \multirow[t]{3}{*}{$\begin{array}{l}\text { Normas/ } \\
\text { Legislação }\end{array}$} & $\begin{array}{l}\text { Existência de legislação atualizada às } \\
\text { necessidades do contexto epidemio- } \\
\text { lógico. }\end{array}$ & 2 & 2 & 2 & 2 & 2 \\
\hline & $\begin{array}{l}\text { Divulgação de normas e portarias para } \\
\text { os municípios da região. }\end{array}$ & 1 & 1 & 1 & 1 & 1 \\
\hline & Total de pontos & $3(100 \%)$ & $3(100 \%)$ & $3(100 \%)$ & $3(100 \%)$ & $3(100 \%)$ \\
\hline \multirow[t]{7}{*}{$\begin{array}{l}\text { Capacidade } \\
\text { Instalada }\end{array}$} & $\begin{array}{l}\text { Quantidade de profissionais de nível } \\
\text { superior suficiente para áreas técnicas. }\end{array}$ & 3 & 0 & 0 & 0 & 0 \\
\hline & $\begin{array}{l}\text { Qualidade técnica dos RH suficiente } \\
\text { para cumprimento das atividades es- } \\
\text { senciais. }\end{array}$ & 3 & 3 & 3 & 0 & 3 \\
\hline & № de salas suficiente. & 2 & 0 & 2 & 0 & 0 \\
\hline & $\begin{array}{l}\text { № de computadores disponíveis e em } \\
\text { funcionamento. }\end{array}$ & 2 & 2 & 2 & 1 & 2 \\
\hline & № de impressoras disponíveis. & 2 & 2 & 2 & 2 & 2 \\
\hline & № de ramais disponíveis. & 2 & 2 & 2 & 2 & 2 \\
\hline & Internet disponível. & 2 & 2 & 2 & 2 & 2 \\
\hline
\end{tabular}


Tabela 1. (cont.)

\begin{tabular}{|c|c|c|c|c|c|c|}
\hline \multirow{4}{*}{$\begin{array}{l}\text { Capacidade } \\
\text { Instalada }\end{array}$} & № veículos disponíveis. & 2 & 2 & 1 & 2 & 1 \\
\hline & $\begin{array}{l}\text { Quantitativo de veículos suficiente em } \\
\text { quantidade e qualidade. }\end{array}$ & 2 & 0 & 0 & 0 & 2 \\
\hline & $\begin{array}{l}\text { Recebimento e distribuição de materiais } \\
\text { e insumos em quantitativo suficiente } \\
\text { para os municípios. }\end{array}$ & 1 & 1 & 1 & 0 & 0 \\
\hline & Total de pontos & $21(100 \%)$ & $14(66,6 \%)$ & $15(71,4 \%)$ & $9(42,8 \%)$ & $14(66,6 \%)$ \\
\hline \multirow[t]{3}{*}{$\begin{array}{l}\text { Recursos } \\
\text { Financeiros }\end{array}$} & $\begin{array}{l}\text { Utilização do repasse da Sevs exclusiva- } \\
\text { mente para as ações de VS. }\end{array}$ & 2 & 0 & 2 & 2 & 2 \\
\hline & $\begin{array}{l}\text { Monitoramento bimestral da execução } \\
\text { do repasse da Sevs. }\end{array}$ & 1 & 0 & 0 & 0 & 1 \\
\hline & Total de pontos & $3(100 \%)$ & $0(0,0 \%)$ & $2(66,6 \%)$ & $2(66,6 \%)$ & $3(100 \%)$ \\
\hline \multirow[t]{4}{*}{$\begin{array}{l}\text { Recursos } \\
\text { Humanos }\end{array}$} & $\begin{array}{l}\% \text { adequado de profissionais qualifi- } \\
\text { cados. }\end{array}$ & 3 & 2 & 0 & 2 & 3 \\
\hline & $\begin{array}{l}\text { Existência de organograma funcional na } \\
\text { região de saúde, incorporando a VS. }\end{array}$ & 1 & 1 & 1 & 1 & 1 \\
\hline & $\begin{array}{l}\text { Disponibilidade de capacitaç̃os/ trei- } \\
\text { namentos de VS para os municípios. }\end{array}$ & 1 & 1 & 1 & 1 & 1 \\
\hline & Total de pontos & $5(100 \%)$ & $4(80,0 \%)$ & $2(40,0 \%)$ & $4(80,0 \%)$ & $5(100 \%)$ \\
\hline \multirow{4}{*}{$\begin{array}{l}\text { Informação } \\
\text { e comuni- } \\
\text { cação }\end{array}$} & $\begin{array}{l}\text { \% de municípios apoiados tecnicamen- } \\
\text { te na elaboração de informes/boletins. }\end{array}$ & 2 & 2 & 0 & 1 & 2 \\
\hline & $\begin{array}{l}\text { Informes e boletins epidemiológicos } \\
\text { elaborados e divulgados mensalmente. }\end{array}$ & 2 & 1 & 2 & 0 & 1 \\
\hline & $\begin{array}{l}\text { № de pautas da CIR com discussão de } \\
\text { temas de VS. }\end{array}$ & 1 & 1 & 1 & 1 & 1 \\
\hline & Total de pontos & $5(100 \%)$ & $4(80,0 \%)$ & $3(60,0 \%)$ & $2(40,0 \%)$ & $4(80,0 \%)$ \\
\hline \multirow[t]{6}{*}{$\begin{array}{l}\text { Vigilância } \\
\text { Laboratorial }\end{array}$} & $\begin{array}{l}\text { Processamento de amostras para diag- } \\
\text { nóstico clínico, ambiental e bromatoló- } \\
\text { gico em nível regional. }\end{array}$ & 2 & 1 & 1 & 2 & 1 \\
\hline & $\begin{array}{l}\text { Amostras provenientes dos municípios } \\
\text { recebidas, armazenadas e transportadas } \\
\text { conforme demanda. }\end{array}$ & 2 & 2 & 2 & 2 & 2 \\
\hline & $\begin{array}{l}\text { Laboratório regional com capacidade } \\
\text { operacional adequada para análise da } \\
\text { qualidade da água. }\end{array}$ & 1 & 0 & 1 & 0 & 1 \\
\hline & $\begin{array}{l}\text { Laboratório regional com capacidade } \\
\text { operacional adequada para sorologias } \\
\text { (dengue, hepatites, chikungunya, doen- } \\
\text { ça de Chagas e leishmaniose). }\end{array}$ & 2 & 1 & 2 & 1 & 1 \\
\hline & $\begin{array}{l}\text { Resultados laboratoriais acompanhados } \\
\text { e divulgados junto aos municípios. }\end{array}$ & 1 & 1 & 1 & 1 & 1 \\
\hline & Total de pontos & $8(100 \%)$ & $5(62,5 \%)$ & $7(87,5 \%)$ & $6(75,0 \%)$ & $6(75,0 \%)$ \\
\hline \multicolumn{2}{|c|}{ Pontuação geral da dimensão estrutura } & $45(100 \%)$ & $30(66,6)$ & $32(71,1)$ & $26(57,7)$ & $35(77,7)$ \\
\hline
\end{tabular}

Fonte: Elaboração própria. 
Tabela 2. Distribuição da pontuação de cada subcomponente da dimensão 'processo' nas regiões I, II, III e IV. Pernambuco, 2017

\begin{tabular}{|c|c|c|c|c|c|c|}
\hline $\begin{array}{l}\text { Subcompo- } \\
\text { nente }\end{array}$ & Indicador & $\begin{array}{r}\text { Pontuação } \\
\text { máxima }\end{array}$ & RI & RII & RIII & RIV \\
\hline \multirow{5}{*}{$\begin{array}{l}\text { Sistemas } \\
\text { de Infor- } \\
\text { mação }\end{array}$} & $\begin{array}{l}\text { Envio/transferência do banco de dados dos SIS } \\
\text { na periodicidade estabelecida. }\end{array}$ & 2 & 2 & 2 & 2 & 2 \\
\hline & $\begin{array}{l}\text { \% de municípios com retroalimentação mensal } \\
\text { dos dados. }\end{array}$ & 2 & 1 & 2 & 1 & 2 \\
\hline & $\begin{array}{l}\text { Análise de dados/indicadores dos municípios, } \\
\text { realizada mensalmente. }\end{array}$ & 2 & 1 & 1 & 1 & 2 \\
\hline & $\begin{array}{l}\text { Assessoramento aos municípios na operaciona- } \\
\text { lização dos SIS conforme demanda. }\end{array}$ & 2 & 2 & 2 & 2 & 2 \\
\hline & Total de pontos & $8(100 \%)$ & $6(75,0 \%)$ & $7(87,5 \%)$ & $6(75,0 \%)$ & $8(100 \%)$ \\
\hline \multirow{3}{*}{$\begin{array}{l}\text { Planeja- } \\
\text { mento }\end{array}$} & Realização de planejamento intersetorial. & 1 & 1 & 1 & 1 & 1 \\
\hline & $\begin{array}{l}\text { Realização de planejamento baseados em } \\
\text { análises de situação de saúde. }\end{array}$ & 1 & 1 & 1 & 1 & 1 \\
\hline & Total de pontos & $2(100 \%)$ & $2(100 \%)$ & $2(100 \%)$ & $2(100 \%)$ & $2(100 \%)$ \\
\hline \multirow{7}{*}{$\begin{array}{l}\text { Integração } \\
\text { (articula- } \\
\text { ção) das } \\
\text { ações }\end{array}$} & $\begin{array}{l}\text { № de reuniões entre as áreas técnicas da VS } \\
\text { regional. }\end{array}$ & 1 & 1 & 1 & 1 & 1 \\
\hline & $\begin{array}{l}\text { № de reuniões entre a VS e a atenção à saúde } \\
\text { regional. }\end{array}$ & 1 & 0 & 0 & 0 & 1 \\
\hline & $\begin{array}{l}\text { Accões conjuntas entre a VS regional e a Sevs, } \\
\text { conforme demanda. }\end{array}$ & 2 & 2 & 2 & 2 & 2 \\
\hline & $\begin{array}{l}\text { \% de municípios assessorados quando as ações } \\
\text { de VS. }\end{array}$ & 2 & 2 & 2 & 2 & 2 \\
\hline & $\begin{array}{l}\text { \% de comitês e/ou grupos técnicos com partici- } \\
\text { pação conjunta (nível central e nível regional). }\end{array}$ & 2 & 0 & 1 & 0 & 1 \\
\hline & $\begin{array}{l}\text { Realização de aç̃oses intersetoriais entre a VS e } \\
\text { outros setores fora da saúde. }\end{array}$ & 1 & 0 & 1 & 1 & 1 \\
\hline & Total de pontos & $9(100 \%)$ & $5(55,5 \%)$ & $7(77,7 \%)$ & $6(66,6 \%)$ & $8(88,8 \%)$ \\
\hline \multirow{3}{*}{$\begin{array}{l}\text { Monito- } \\
\text { ramento e } \\
\text { avaliação }\end{array}$} & $\begin{array}{l}\text { Monitoramento trimestral dos indicadores de } \\
\text { VS pela equipe regional. }\end{array}$ & 2 & 0 & 2 & 1 & 1 \\
\hline & $\begin{array}{l}\text { Existência de reuniões para a discussão dos re- } \\
\text { sultados do monitoramento com os municípios. }\end{array}$ & 1 & 1 & 1 & 1 & 1 \\
\hline & Total de pontos & $3(100 \%)$ & $1(33,3 \%)$ & $3(100 \%)$ & $2(66,6 \%)$ & $2(66,6 \%)$ \\
\hline \multirow{5}{*}{$\begin{array}{l}\text { Desenvol- } \\
\text { vimento } \\
\text { das ações } \\
\text { de VS }\end{array}$} & $\begin{array}{l}\text { VS da regional estruturada para detecção e } \\
\text { resposta aos eventos de importância para saúde } \\
\text { pública. }\end{array}$ & 2 & 0 & 2 & 0 & 1 \\
\hline & $\begin{array}{l}\text { Análises epidemiológicas realizadas e divul- } \\
\text { gadas aos municípios mensalmente. }\end{array}$ & 2 & 1 & 0 & 0 & 2 \\
\hline & $\begin{array}{l}\text { \% de municípios com apoio técnico da VS re- } \\
\text { gional na investigação epidemiológica de casos } \\
\text { notificados, surtos e óbitos, conforme demanda. }\end{array}$ & 2 & 2 & 2 & 2 & 2 \\
\hline & $\begin{array}{l}\text { \% de municípios com apoio técnico da VS } \\
\text { regional para ações de Promoção, Prevenção } \\
\text { e Controle de doenças e agravos, conforme } \\
\text { demanda. }\end{array}$ & 2 & 2 & 2 & 2 & 2 \\
\hline & Total de pontos & $8(100 \%)$ & $450,0 \%)$ & $675,0 \%)$ & $4(50,0 \%)$ & $7(87,5 \%)$ \\
\hline Pontuação \&̊ & geral da dimensão processo & $30(100 \%)$ & $18(60 \%)$ & $25(83,3 \%)$ & $20(66,6 \%)$ & $27(90 \%)$ \\
\hline
\end{tabular}

Fonte: Elaboração própria. 
Observa-se que o único subcomponente que atingiu $100 \%$ de implantação nas quatro regiões investigadas foi 'Normas/Legislação', que englobou a existência de legislação atualizada às necessidades do contexto epidemiológico, bem como a divulgação de normas e portarias para os municípios da região. Vale ressaltar também que as regiões apresentaram como 'implantado' os seguintes aspectos relacionados à 'estrutura': estrutura física relativa a impressoras, ramais, internet disponíveis; existência de organograma funcional incorporando a VS; disponibilidade de capacitação e treinamento para os municípios; pelo menos uma pauta na Comissão Intergestores Regional (CIR) com discussão de temas de VS; recebimento, armazenamento e transporte de amostras laboratoriais proveniente dos municípios de acordo com a demanda; e acompanhamento e divulgação, junto aos municípios, dos resultados laboratoriais (tabela 1).

No que diz respeito ao ponto crítico da dimensão 'estrutura', pode-se destacar no subcomponente 'capacidade instalada', a insuficiência de profissionais de nível superior para áreas técnicas, que apresentou o percentual zero em todas as regiões estudadas. Além disso, apenas a região II fez referência ao número de salas suficiente (100,0\%), e somente a RIV ao quantitativo de veículos suficiente em quantidade e qualidade (100,0\%). Com relação ao subcomponente 'recursos financeiros', estava 'implantado' somente na região IV quanto ao quesito 'monitoramento bimestral da execução do repasse da Sevs' (100,0\%) (tabela 1).

As regiões II e IV apresentaram desempenho superior na dimensão 'processo' em relação à dimensão estrutura, com os melhores resultados para os subcomponentes 'sistemas de informação' (87,5\% e 100,0\%) e 'planejamento' $(100,0 \%$ e $100,0 \%)$. Em contrapartida, as regiões I e III destacaram-se negativamente nos subcomponentes 'integração das ações' (55,5\% e $66,6 \%)$, 'monitoramento e avaliação' (33,3\% e $66,6 \%$ ) e 'desenvolvimento das ações de VS' (50,0\% e 50,0\%) (tabela 2).

Destaca-se que alguns quesitos foram considerados 'implantados' nas quatro regiões estudadas, como envio e transferência do banco de dados dos SIS na periodicidade estabelecida; assessoramento aos municípios na operacionalização dos SIS; realização de planejamento intersetorial; realização de planejamento baseados em análises de situação de saúde; realização de reuniões entre as áreas técnicas da VS regional; realização de ações conjuntas entre a VS regional e a Sevs; assessoramento aos municípios nas ações de VS; realização de reuniões para a discussão dos resultados do monitoramento com os municípios; apoio técnico aos municípios na investigação epidemiológica de casos notificados, surtos e óbitos; e apoio técnico aos municípios nas ações de promoção, prevenção e controle de doenças e agravos.

Com relação às atividades mais críticas, citam-se a realização de reuniões entre a VS e a atenção à saúde, em que apenas a região IV apresentou resultado favorável; a estruturação da VS regional para detecção e resposta aos eventos de importância para saúde pública, em que apenas a região II atingiu resultado satisfatório; e a realização de análises epidemiológicas com divulgação aos municípios mensalmente, em que apenas a região IV apresentou resultado favorável.

\section{A percepção dos gestores com relação ao processo de regionalização da VS}

A análise das entrevistas permitiu observar que a percepção dos gestores dos níveis central e regional foi complementar e congruente. O GC1 afirmou que o processo de regionalização da VS se deu a partir da construção do Plano Diretor de Regionalização (PDR) de 2011, com a criação das doze regiões de saúde, as quais passaram a ter coordenação e gerência de vigilância responsáveis pela condução e realização das ações no nível local. O GC3 complementou que esse processo seguiu direcionamentos distintos, de acordo com a gestão estadual vigente. Esse mesmo gestor também afirmou que, a partir de 2011, o estado passou a investir em apoiadores para as regionais que, a partir de então, passaram a ter posicionamento mais efetivo na Secretaria Estadual 
de Saúde (SES), uma vez que nada pode ser repassado diretamente aos municípios.

A fala do GC2, entretanto, revelou que essa realidade se deu de forma desigual entre as regiões de saúde, pois a estrutura de algumas delas era bastante precária. Ele destacou que, inicialmente, o principal papel das regionais era de ponto de contato do nível central com os municípios, em vez de exercer a função de gestão e coordenação do sistema de VS no nível regional, para a qual foram criadas.

Todos os gestores entrevistados consideraram a regionalização da VS muito importante. $\mathrm{O}$ GCl afirmou que a região surgiu a partir da necessidade de pensar estratégias para resolver os problemas de VS, adequadas a cada contexto, dadas as diferentes realidades dos municípios. Além disso, enfatizou que a regionalização contribui para a organização da rede de serviços a partir das necessidades do território, tendo em vista que ainda existe alta concentração de serviços na região metropolitana de Recife devido à conformação histórica do estado de Pernambuco.

Para o GC2, a regionalização é importante, porque, devido ao desenho territorial do estado, a atuação apenas do nível central seria insuficiente para que todas as ações ocorressem de forma articulada com os municípios. Para ele, como a descentralização foi incompleta em alguns municípios, a regional funciona como um impulsionador das ações sejam realizadas. Nesse sentido, o GC3 afirma que "não existe VS sem regionalização", pois a resolução de determinados problemas de maneira centralizada torna-se pouco efetiva e inoportuna.

Com relação às potencialidades, o $\mathrm{GCl}$ e o GR4 mencionaram a questão normativa. O GR4 complementou ao relatar que a legislação impõe obrigações para as regiões de saúde e para os municípios, além de despertar nos gestores a necessidade do trabalho conjunto, à medida que "o fluxo das doenças e dos serviços entre os municípios é muito grande".

O GCl, assim como o GR3 e o GR4, compartilhou que a conformação atual das regiões de saúde, baseada na geografia territorial e na situação de saúde, facilita a regionalização à medida que o nível regional pode dar maior assistência aos municípios, além de possibilitar a discussão da situação de saúde nos locais onde acontecem agravos com maior frequência. Outro ponto destacado, dessa vez pelos GC2, GRl e GR4, diz respeito ao apoio técnico do nível central a partir da contratação de apoiadores institucionais para atuar nas regionais, além da disponibilidade de equipamentos, bem como da realização de capacitações, treinamentos e reuniões sempre que necessário. O GC3 também ressaltou esse ponto e ainda relatou que a gestão da VS no nível central da SES-PE tem favorecido o processo de regionalização, visto que, desde 2011, o estado tem investido em recursos financeiros, equipamentos, veículos e na contratação de pessoal.

Outro aspecto é a existência do colegiado de gestores regionais, o que acontece com a presença de técnicos e gestores do nível central da VS da SES, bem como de coordenadores de VS de todas as regiões de saúde. De acordo com o GR4, essas reuniões incentivam os municípios a elencar pautas para discussão em comum, ao passo que o compartilhamento e a proposição de ações conjuntas contribuem para o fortalecimento das ações e melhoria da situação de saúde.

Quanto às fragilidades, $\mathrm{o}$ aspecto mencionado pela totalidade dos gestores entrevistados diz respeito à deficiência na capacidade técnica dos profissionais da VS nas regiões de saúde. Para o GR1, GR4 e o GC2, há carência de profissionais com perfil e conhecimento para trabalhar na VS, o que se torna ainda mais evidente em algumas regiões específicas. Para o GR3, outra dificuldade relacionada aos recursos humanos está nos contratos dos apoiadores institucionais, por serem firmados de forma temporária, o que constitui uma barreira para a continuidade do serviço.

Além disso, o GC1 afirmou que a falta de autonomia técnica e financeira, além da insuficiência de mecanismos que favoreçam a pactuação entre as regiões, compromete o processo de regionalização. Na percepção do GR2, os municípios também não possuem autonomia técnica e apenas cumprem metas, agindo de maneira pontual à demanda da instância estadual. 


\section{Discussão}

A VS regionalizada em Pernambuco mostrou-se parcialmente implantada. As variações entre as regiões e entre as dimensões 'estrutura' e 'processo' estão, no geral, coerentes com as falas dos gestores entrevistados. Apesar do reduzido número de estudos relacionados à avaliação no nível regional, a estratégia metodológica utilizada nesta pesquisa permitiu abordar as principais questões para avaliação da VS nesse nível, uma vez que se baseou em subcomponentes e indicadores previamente validados por especialistas da área de VS. Mensurar a eficiência de sistemas regionais é de complexa realização devido a suas características particulares, especialmente aos recursos socioeconômicos disponíveis ${ }^{19}$.

Este estudo contém limitações importantes, uma vez que não foi possível realizar a análise de implantação nas doze regiões de saúde que compõem o arranjo da regionalização em Pernambuco. Alternativamente, decidiu-se pela realização nas quatro regiões de saúde que se apresentam como sede das macrorregionais. Além disso, outro fator limitante foi selecionar apenas os gestores para entrevista, deixando de considerar os técnicos que trabalham na VS e suas respectivas percepções.

A implantação da VS regionalizada foi definida a partir da análise da estrutura dos serviços e do processo de trabalho, o que permitiu revelar diferenças entre as regiões de saúde. Os achados deste estudo contêm dificuldades na dimensão 'estrutura', com destaque para o número insuficiente de profissionais de nível superior. Outros estudos também constataram a inexistência de categorias profissionais fundamentais para o desenvolvimento de todas as ações técnicas e de gestão da VS, assim como a prevalência de trabalhadores de nível médio em detrimento do número de profissionais de nível superior. Identificaram, ainda, elevado número de trabalhadores sem formação específica para o trabalho ${ }^{\mathbf{7} 20}$. Neste estudo, três gestores afirmaram haver carência de profissionais com perfil e conhecimento adequados à atuação na VS. Para Donateli21, a existência de recursos humanos com formação inadequada favorece a atuação incipiente da VS.

No que diz respeito ao subcomponente 'capacidade instalada', em apenas uma das regiões se constatou a presença de veículos em quantidade e qualidade suficientes para atender à demanda. Outros estudos também abordam a disponibilidade de veículos como ponto crítico $^{22,23}$. A insuficiência de veículos é um aspecto que dificulta o processo de trabalho e impede a realização de várias atividades inerentes à vigilância ${ }^{22}$.

Ressalta-se como um ponto positivo revelado neste estudo a percepção dos gestores quanto à existência de uma legislação atualizada para VS, sendo esse item indicado como uma das potencialidades para o processo de regionalização. No entanto, é importante destacar que, embora a VS possua um amplo leque normativo, a legislação específica definindo atribuições aos diversos níveis do sistema e estratégias para o desenvolvimento da regionalização ainda é precária, 5,24.

Contudo, é possível observar um movimento no sentido da reorganização das ações de VS após a aprovação da primeira Política Nacional de Vigilância à Saúde (PNVS) durante a I Conferência Nacional de Vigilância em Saúde (CNVS). A PNVS foi instituída por meio da Resolução nº 588 , de 18 de julho de 2018, e compõe um conjunto de diretrizes que orientam as práticas de promoção e proteção de doenças e agravos nas diversas áreas da VS. Ela reforça a necessidade de articulação entre as vigilâncias e a atenção em saúde por meio de serviços articulados no âmbito da região de saúde como uma estratégia para a organização da VS 25 .

Este estudo também revelou dificuldades na dimensão 'processo', visto que apenas uma região atingiu a pontuação máxima no indicador 'análise mensal dos dados/indicadores dos municípios'. Entretanto, é importante destacar que o conhecimento e a análise da situação de saúde são fundamentais para o planejamento das ações de vigilância, que deve estabelecer prioridades e indicadores para 
*Orcid (Open Researcher and Contributor ID). acompanhamento e avaliação dessas ações 26-28 .

Quanto à incipiência do número de reuniões entre a VS e a atenção à saúde no nível regional, a Portaria $n^{\circ} 1.378$, de 9 de julho de 2013, que regulamenta as responsabilidades e define diretrizes para execução e financiamento das ações de VS pelos diferentes entes da federação, destaca a importância da integração dessas áreas por meio do desenvolvimento de processo de trabalho que reflita a realidade local ${ }^{15}$. Nesse sentido, estudo realizado sobre a VS no espaço das práticas da atenção básica relatou a importância de a equipe de saúde local se reconhecer como agente de vigilância. A finalidade é distinguir os riscos aos quais a população está exposta e, assim, promover a integração de práticas de saúde por meio da percepção ampliada das necessidades do território ${ }^{29}$.

Por oportuno, cabe destacar que a Portaria $\mathrm{n}^{\circ} 3.992$, de 28 de dezembro de 2017 , reduziu de seis para dois os blocos de financiamento do SUS, sendo um de custeio e outro de investimentos, excluindo o bloco da VS e flexibilizando do uso das verbas. Essa mudança poderá significar menos investimento em VS, à medida que os estados e municípios passarão a definir onde investir os repasses feitos pelo Ministério da Saúde (MS) ${ }^{30}$.

\section{Conclusões}

Apesar de a regionalização da VS em Pernambuco ainda estar incompleta e de os resultados deste estudo demonstrarem variações significativas entre as regiões estudadas no que diz respeito à estrutura disponível e ao processo de trabalho, é importante destacar a relevância do processo, uma vez que o nível regional pode planejar e promover estratégias mais adequadas a cada contexto, observando as diferentes realidades dos municípios.

Falhas estruturais e gestão dos recursos humanos permanecem como impedimento para regionalização das ações e serviços de VS, bem como a integração das ações de prevenção e controle com a atenção básica, alinhando-as aos demais serviços da rede de atenção. Mesmo que o acompanhamento longitudinal das pessoas exista na atenção primária, a continuidade do cuidado pode implicar intervenções diagnósticas e tratamentos de média e alta complexidade frequentemente indisponíveis na maioria dos municípios. Assim, a atuação do nível estadual, tanto no desenho da rede de prestação de serviços como no estabelecimento de mecanismos para sua integração, é condição imprescindível ao sucesso de programas de controle dos agravos e constitui importante desafio para o avanço do processo de regionalização da VS.

Por fim, espera-se que os achados deste estudo possam contribuir para a discussão sobre a regionalização da VS no Brasil, pois suas fragilidades são claras e indicam a necessidade do princípio da solidariedade, que vai além do atendimento aos preceitos legais e normativos; de políticas públicas mais efetivas; e de lideranças institucionais, a serem exercidas pelos estados. Os gestores estaduais precisam assumir o protagonismo na indução da regionalização como projeto de governo, pois a capacidade de articulação do gestor estadual em promover o envolvimento dos atores e favorecer o pertencimento regional é uma tarefa complexa, mas é, sem dúvida, crucial para o desenvolvimento de uma regionalização cooperativa ${ }^{31}$.

\section{Colaboradores}

Lopes NB (0000-0003-3159-5425)* contribuiu substancialmente para concepção, planejamento, análise e interpretação dos dados; elaboração do rascunho e revisão crítica do conteúdo; aprovação da versão final do manuscrito. Albuquerque AC (0000-0001-63054127)* e Felisberto E (0000-0002-2316-2251)* contribuíram para concepção e planejamento da pesquisa, bem como aprovação da versão final do manuscrito. 


\section{Referências}

1. Brasil. Casa Civil. Lei no $8.080 / 90$, de 19 de setembro de 1990. Dispõe sobre as condições para promoção, proteção e recuperação da saúde, a organização e o funcionamento dos serviços correspondentes e dá outras providências. Diário Oficial da União. 19 Set 1990. Brasília, DF. Seção 1:18.055.

2. Oliveira SRA, Teixeira CF. Avaliação da Regionalização do SUS: Construção do modelo teórico-lógico. RBSP. 2013; 37(1):236-254.

3. Rolim LB, Cruz RSBLC, Sampaio KJAJ. Participação popular e o controle social como diretriz do SUS: uma revisão narrativa. Saúde debate. 2013;37(96):139-147.

4. Lima LD, Queiroz LFN, Machado CV, et al. Descentralização e regionalização: dinâmica e condicionantes da implantação do Pacto pela Saúde no Brasil. Ciênc. Saúde Colet. 2012; 17(7):1903-1914.

5. Vargas I, Mogollón-Perez AS, Unger JP, et al. Regional-based Integrated Healthcare Network policy in Brazil: from formulation to practice [internet]. Health PolicyPlann. 2014 [acesso em 2018 ago 15]; 30(6):70517. Disponível em: https://www.ncbi.nlm.nih.gov/ pubmed/24963157.

6. Brasil. Casa Civil. Decreto $n^{0} 7.508$, de 28 de junho de 2011. Regulamenta a Lei no 8.080 , de 19 de setembro de 1990. Diário Oficial da União. 28 Jun 2011. Brasília, DF. Seção 1:1.

7. Albuquerque AC, Cesse EAP, Felisberto E, et al. Regionalização da vigilância em saúde: uma proposta de avaliação de desempenho em uma região de saúde no Brasil. Rev. Bras. Saúde Mater. Infant. [internet]. 2017 [acesso em 2018 ago 15]; 17(supl1):S135-S151. Disponível em: http://www.scielo.br/scielo.php?script=sci_ arttext\&pid=S1519-38292017000600007\&lng=en\&n $\mathrm{rm}=\mathrm{iso} \& \operatorname{lng}=\mathrm{pt}$.

8. Faria LS, Bertolozi MR. Aproximações teóricas acerca da Vigilância em Saúde: um horizonte para a integralidade. Acta Paul Enferm. 2009; 22(4):422-7.
9. Oliveira AEF, Reis RS. Gestão Pública em Saúde. Os desafios da avaliação em Saúde. São Luiz: EDUFMA; 2016.

10. Donabedian A. Evaluating the quality of medical care. Milbank Memorial Fund. Milbank Q. 2005; 83(4):691729.

11. Pernambuco. Secretaria Executiva de Regulação em Saúde. Plano Diretor de Regionalização. Recife: Secretaria de Saúde do Estado de Pernambuco; 2011.

12. Brasil. Ministério da Saúde. Portaria $\mathrm{n}^{0} 1.399$, de 15 de dezembro de 1999. Regulamenta a NOB SUS 01/96 no que se refere às competências da União, estados, municípios e Distrito Federal, na área de epidemiologia e controle de doenças, define a sistemática de financiamento e dá outras providências. Diário Oficial da União. 15 Dez 1999. Brasília, DF. Seção 1.

13. Brasil. Ministério da Saúde. Portaria $\mathrm{n}^{\circ} 1.378$, de 9 de julho de 2013. Regulamenta as responsabilidades e define diretrizes para execução e financiamento das ações de Vigilância em Saúde pela União, Estados, Distrito Federal e Municípios, relativos ao Sistema Nacional de Vigilância em Saúde e Sistema Nacional de Vigilância Sanitária. Diário Oficial da União. 9 Jul 2013. Brasília, DF. Seção 1:48. Seção 1:61. [acesso em 2017 abr 10]. Disponível em: http://www.saude. sp.gov.br/resources/ccd/homepage/acesso-rapido/ civs/portaria_20_de_03_outubro.pdf.

14. Brasil. Ministério da Saúde. Secretaria de Vigilância à Saúde. Secretaria de Atenção à Saúde. Diretrizes Nacionais da Vigilância em Saúde. Comunicação e Educação em Saúde. Brasília, DF: Ministério da Saúde; 2010. (Série F. Comunicação e Educação em Saúde) (Série Pactos pela Saúde 2006; v. 13).

15. Brasil. Ministério da Saúde. Portaria n ${ }^{0} 20$, de 03 de outubro de 2003. Regulamenta a coleta de dados, fluxo e periodicidade de envio das informações sobre óbitos e nascidos vivos para os Sistemas de Informações em Saúde - SIM e Sinasc. Diário Oficial da União. 3 Out 2003. Brasília, DF. 
16. Brasil. Ministério da Saúde. Instrução Normativa $n^{0}$ 02 de 22 de novembro de 2005. Regulamenta as atividades da vigilância epidemiológica com relação à coleta, fluxo e a periodicidade de envio de dados da notificação compulsória de doenças por meio do Sistema de Informação de Agravos de Notificação - SINAN. [acesso em 2017 abr 10]. Disponível em: http://bvsms.saude.gov.br/bvs/saudelegis/svs/2005/ int0002_22_11_2005.html.

17. Bardin L. Análise de conteúdo. São Paulo: Edições $70 ; 2011$.

18. Minayo MCS. O desafio do conhecimento: pesquisa qualitativa em saúde. São Paulo: Hucitec; 2015.

19. Aristovnik A. Regional Performance Measurement of Healthcare Systems in the EU: A Non-parametric Approach. Lex Localis. 2015 [acesso em 2017 out 30]; 13(3):579-593. Disponível em: https://doi. org/10.4335/13.3.579-593(2015).

20. Mendes TKA, Oliveira SP, Delamarque EV, et al. Reestruturação da Gestão das Vigilâncias em Saúde em Alagoas: A Precarização da Formação e do Trabalho. Trab. educ. saúde [internet]. 2016 [acesso 2017 out 25]; 14(2):421-443. Disponível em: http://www. scielo.br/scielo.php?script=sci_arttext\&pid=S1981$-77462016000200421 \& \operatorname{lng}=$ pt\&nrm=iso.

21. Donateli CP, Avelar OS, Einloft ABN, et al. Avaliação da Vigilância em Saúde na Zona da Mata Mineira, Brasil: das normas à prática. Ciênc. Saúde Colet. 2017; 22(10):3439-3455.

22. Albuquerque AC, Mota ELA, Felisberto E. Descentralização das ações de vigilância epidemiológica em Pernambuco, Brasil. Cad. Saúde Pública. 2015; 31(4):861-873.

23. Costa CS, Rocha AM, Silva GS, et al. Programa de Controle da Esquistossomose: avaliação da implantação em três municípios da Zona da Mata de Pernambuco, Brasil. Saúde debate. 2017; 41(esp):229-241.
24. Bezerra LCA, Freese E, Frias PG, et al. A vigilância epidemiológica no âmbito municipal: avaliação do grau de implantação das ações. Cad. Saúde Pública. 2009; 25(4):827-839.

25. Conselho Nacional de Saúde. Resolução 588, de 12 de julho de 2018. [acesso em 2018 ago 14]. Disponível em: http://conselho.saude.gov.br/resolucoes/2018/ Reso588.pdf.

26. Costa JMBS, Cesse EAP, Samico IC, et al. Avaliação do desempenho estadual da vigilância em saúde de Pernambuco. Physis. 2015; 25(4):1141-63.

27. Costa ICS, Souto AC. Indicadores em Vigilância Sanitária de Alimentos. Revista Baiana de Saúde Pública. 2011; 35(4):826-844.

28. Weigelt LD, Mancio JG, Petry ELS. Indicadores de saúde na visão dos gestores dos municípios no âmbito da $13^{\text {a }}$ Coordenadoria Regional de Saúde - RS. 1. 2012; (36):191-205.

29. Oliveira CM, Casanova AO. Vigilância da saúde no espaço de práticas da atenção básica. Ciênc. Saúde Colet. 2009; 14(3): 929-936.

30. Brasil. Ministério da Saúde. Portaria n ${ }^{0}$ 3992, d 28 de dezembro de 2017. Altera a Portaria de Consolidação $\mathrm{n}^{\circ}$ 6/GM/MS, de 28 de setembro de 2017, para dispor sobre o financiamento e a transferência dos recursos federais para as ações e os serviços públicos de saúde do Sistema Único de Saúde. [acesso em 2018 ago 14]. Disponível em: http://bvsms.saude.gov.br/bvs/ saudelegis/gm/2017/prt3992.

31. Reis YAC, Cesse E A P, Carvalho EF. Consensos sobre o papel do gestor estadual na regionalização da assistência à saúde no Sistema Único de Saúde (SUS). Rev. Bras. Saúde Matern Infant. 2010; 10 (supl1):157-72.

Recebido em 11/09/2018

Aprovado em 15/05/2019

Conflito de interesses: inexistente

Suporte financeiro: não houve 